\title{
TELEOPERACIÓN DE INSTRUMENTOS QUIRÚRGICOS ARTICULADOS
}

\author{
A. M. Gómez Delgado, C.J. Pérez del Pulgar Mancebo, A. Reina Terol, V.F. Muñoz \\ Departamento de Ingeniería de Sistemas y Automática, Universidad de Málaga, Andalucia Tech \\ carlosperez@uma.es
}

\begin{abstract}
Resumen
En los últimos años se han llevado a cabo grandes avances en el ámbito de la robótica médica que han dado lugar al estudio y desarrollo de diversos robots quirúrgicos. En particular, la cirugía minimamente invasiva ha evolucionado hacia lo que se denomina cirugía de un único puerto. Este tipo de cirugía permite introducir todos los instrumentos a través de un único punto de inserción. En este sentido, en este trabajo se describe la plataforma CISOBOT, desarrollada por la Universidad de Málaga para este tipo de intervenciones, así como su ampliación a través del diseño mecatrónico de un instrumento motorizado que permite mover la punta de éste. El objetivo de esta plataforma es el estudio de nuevos algoritmos de control que permitan la teleoperación bilateral y el empleo de guiado háptico.
\end{abstract}

Palabras clave: Teleoperación, robótica médica, instrumento motorizado, dispositivo háptico

\section{INTRODUCCIÓN}

En los últimos años, se han realizado grandes avances relacionados con las técnicas quirúrgicas basadas en cirugía minimamente invasiva (MIS). El estudio y desarrollo de técnicas SILS (cirugías de un solo puerto por sus siglas en inglés) han permitido introducir varios instrumentos articulados a través de un mismo punto de inserción (fulcro). Algunos ejemplos son: cirugía por una sola incisión (SPAS), laparoscopia por una sola incisión (LESS) o cirugía endoscópica transluminal a través de orificios naturales (NOTES) [3].

Este tipo de procedimientos aportan ventajas al paciente como: disminución del malestar, riesgo de hemorragias y complicaciones, debido al menor trauma causado por el menor número de incisiones. Además, el tiempo de recuperación se ve también disminuido por este mismo motivo [5].

Sin embargo, estos beneficios derivan en nuevos inconvenientes para el cirujano. Se debe tener en cuenta el posible choque entre instrumentos tanto fuera como dentro del abdomen del paciente de-

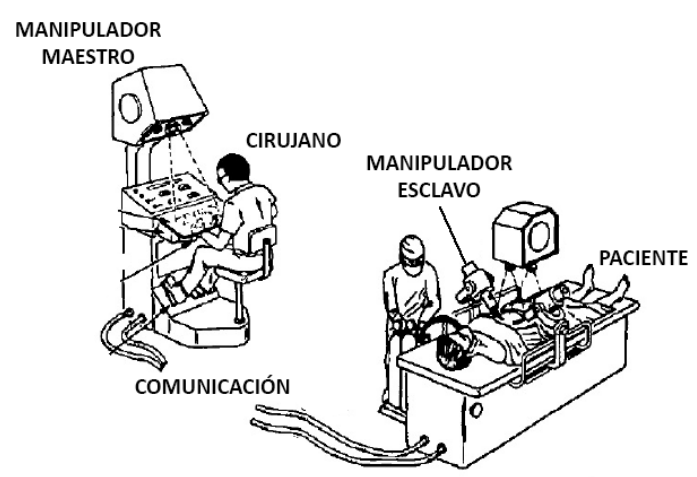

Figura 1: Ejemplo de sistema de teleoperación en cirugía.

bido a la introducción de varios instrumentos a través del mismo trocar. Además, la cámara laparoscópica también se introduce por la misma incisión, provocando una pérdida de triangulación y una reducción del campo de visión dentro del abdomen debido a su proximidad a los instrumentos [8].

El empleo de plataformas robóticas de teleoperación se emplean en cirugía para mejorar las habilidades del cirujano en intervenciones quirúrgicas [10]. Estas plataformas se basan en una arquitectura maestro esclavo tal y como que muestra en la Figura 1. El cirujano envía las órdenes mediante una consola (maestro) dotada normalmente de una pantalla y dispositivos hápticos. Estas órdenes son recibidas por uno o varios manipuladores que son los encargados de reproducir los movimientos del cirujano.

El empleo de estas plataformas aparece como una solución que podría apaliar las desventajas explicadas anteriormente, se podría disponer de un sistema capaz de detectar y esquivar la colisión de instrumentos, control del movimiento de los instrumentos con el fin de facilitar la ejecución de estos, etc. En este sentido, la Universidad de Málaga se encuentra desarrollando la plataforma experimental de teleoperación CISOBOT [2] [7]. Este artículo contribuye al desarrollo de esta plataforma con el diseño mecatrónico de un instru- 


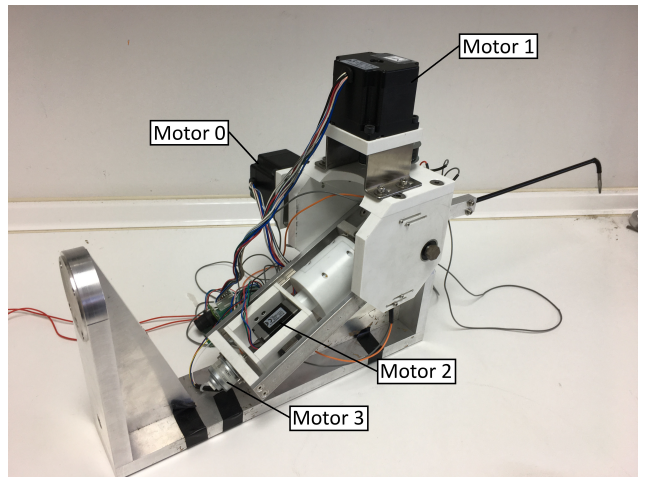

Figura 2: Instrumento motorizado con punta articulada.

mento motorizado que permite mover la punta y el control del mismo mediante una consola de teleoperación que emplea dispositivos hápticos.

\section{INSTRUMENTO MOTORIZADO}

El prototipo del instrumento motorizado con punta articulada utilizado es el representado en la Figura 2. Este instrumento ha sido diseñado y fabricado en colaboración con la empresa PROSAIN S.L. El objetivo de esta herramienta es añadir tres grados de libertad en la punta de un instrumento quirúrgico empleado para técnicas SILS.

Dicho instrumento consta de una pinza en el extremo, cuatro motores paso a paso y cuatro finales de carrera. De estos, tres motores van conectados a una controladora Trinamic TMCM-351, y el motor encargado de la apertura y cierre de la pinza va conectado a la controladora de Nanotec SMC42. A continuación, se explica con detalle dichos componentes.

\section{$2.1 \quad$ MOTORES PASO A PASO}

Los motores paso a paso se encargan de transmitir el movimiento a la punta articulada a través de cables tensores distribuidos a lo largo de la varilla. Esto proporciona al instrumento tres grados de libertad del tipo Roll-Pitch-Yaw. Este tipo de motores avanzan girando a pasos fijos de $1,8^{\circ}$, sin embargo, al estar conectado a un sistema de poleas, el ángulo por paso queda en $0,9^{\circ}$.

Siguiendo la numeración expuesta en la figura 2, el Motor 0 realiza el movimiento vertical de la punta, coincidiendo con la rotación denominada Pitch, el Motor 1 realiza el movimiento horizontal, que se corresponde con la rotación Yaw, y por último, el Motor 2 se encarga del movimiento de la varilla del instrumento sobre su propio eje, coincidiendo con la rotación denominada Roll. De este modo, se da

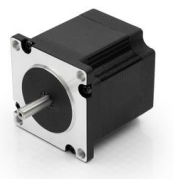

(a) Motor 0 y 1

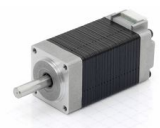

(b) Motor 2

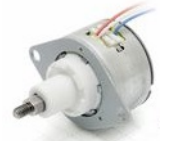

(c) Motor 3
Figura 3: Motores paso a paso empleados en el instrumento motorizado.

capacidad a la punta de moverse en las tres direcciones espaciales. Los tres motores, del fabricante Nanotec, son los mostrados en la figura 3a y 3b, y sus características más relevantes se describen en las tablas 1 y 2 .

Tabla 1: Características de los motores 0 y 1

\begin{tabular}{|l|c|}
\hline Especificación & Motor 0 y 1 \\
\hline Modelo & ST5918S2008-A \\
Voltaje & $3.0 \mathrm{~V}$ \\
Corriente/Fase & $2.0 \mathrm{~A}$ \\
Par de mantenimiento & $88 \mathrm{Ncm}$ \\
Peso & $0.65 \mathrm{Kg}$ \\
\hline
\end{tabular}

Tabla 2: Características del Motor 2

\begin{tabular}{|l|c|}
\hline Especificación & Motor 2 \\
\hline Modelo & ST2018M0804-A \\
Voltaje & $4.32 \mathrm{~V}$ \\
Corriente/Fase & $0.8 \mathrm{~A}$ \\
Par de mantenimiento & $3.00 \mathrm{Ncm}$ \\
Peso & $0.08 \mathrm{Kg}$ \\
\hline
\end{tabular}

La función de abrir y cerrar la pinza que se encuentra en el extremo del instrumento la lleva a cabo el cuarto motor (Figura 3c), denominado Motor 3 en la figura 2. El movimiento rotativo se convierte en un movimiento lineal por la tuerca de rosca integrada en el motor lineal paso a paso, comportándose como un actuador lineal. En la tabla 3 se encuentran resumidas las principales características de dicho motor.

\subsection{CONTROLADORAS}

El esquema de conexiones realizado en la interfaz entre las controladoras y el instrumento motorizado se muestra en la figura 4. Para mayor claridad, se ha seguido el mismo código de colores de las conexiones en las placas reales.

La controladora TMCM-351 (Figura 5a) se encarga de realizar el control en tiempo real de los motores paso a paso del instrumento. Este modelo es capaz de controlar precisamente tres motores. Según la información del fabricante, para la ali- 


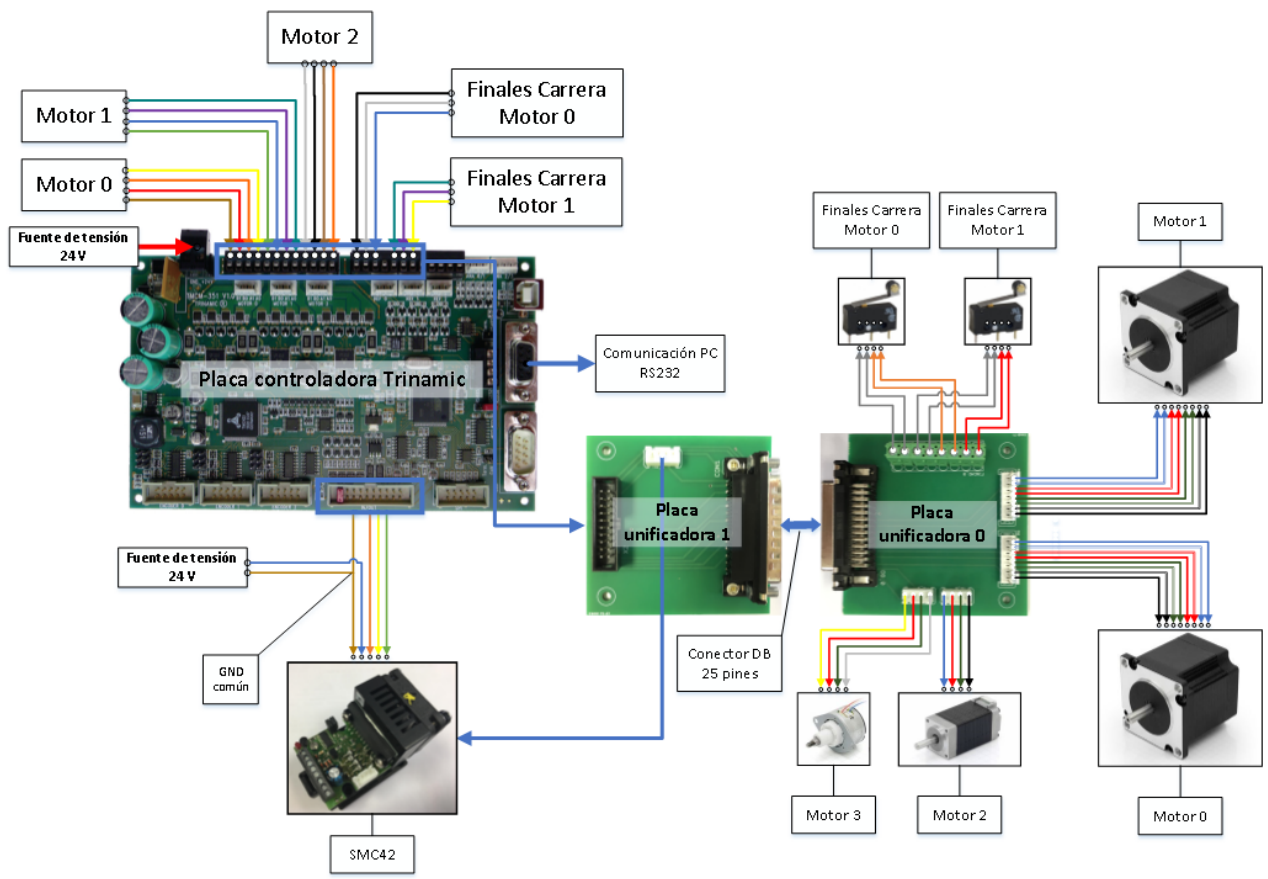

Figura 4: Esquema de conexionado entre las controladoras y los motores.

Tabla 3: Características del Motor 3

\begin{tabular}{|l|c|}
\hline Especificación & Motor 3 \\
\hline Modelo & LPV2515S0104-Tr3.5X1 \\
Voltaje & $5.0 \mathrm{~V}$ \\
Corriente/fase & $0.1 \mathrm{~A}$ \\
Carrera & $12 \mathrm{~mm}$ \\
Peso & $0.04 \mathrm{Kg}$ \\
\hline
\end{tabular}

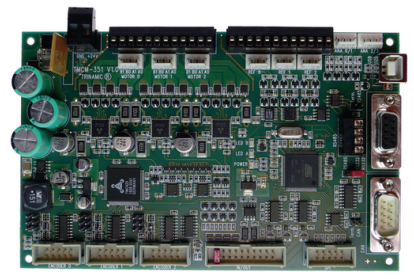

(a) TMCM-351

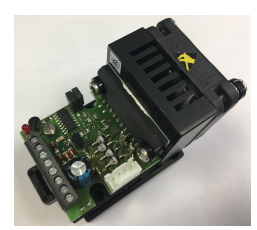

(b) $\mathrm{SMC} 42$
Figura 5: Controladoras empleadas.

mentación será necesaria una tensión continua de $24 \mathrm{~V}$, y una corriente máxima de $2,8 \mathrm{~A}$ por cada motor. Finalmente, la placa se comunica con el $\mathrm{PC}$ a través de una conexión serie RS-232.

El control del Motor 3 se lleva a cabo por medio de la controladora SMC42 de la empresa Nanotec, mostrada en la figura 5b. Sus características principales son: voltaje de funcionamiento entre $21 \mathrm{~V}$ y $37 \mathrm{~V}$ a corriente continua y una capacidad de corriente máxima de $2 \mathrm{~A}$ por fase. Se encuentra conectada a la placa controladora TMCM-351, empleando las señales Direction, Enable y Clock para controlar el movimiento del motor 3. Cada paso se ejecuta mediante un flanco negativo en la entrada Clock. La señal Direction especifica la dirección de rotación del motor. Por último, la señal Enable desconecta la corriente de fase después de recibir un flanco de subida y el motor no recibiría corriente. La señal de un flanco de bajada suministra el motor con corriente de nuevo. De esta forma, la señal Enable debe estar a nivel bajo para realizar un movimiento del motor.

Cada motor transmite el movimiento a la punta a través de unos cables tensores en el interior de la varilla, lo cuál provoca que la relación entre el movimiento del motor y la posición de la punta no sea una relación lineal. Por este motivo, se ha desarrollado un programa encargado de calibrar la relación motor-instrumento.

La inicialización de los motores se realiza mediante el empleo de los cuatro finales de carrera instalados en el instrumento. Con ellos se obtienen las referencias del Motor 0 y el Motor 1 y se inicializa de manera que la punta quede recta. Además, se configuran las variables Clock, Direction y Enable, necesarias para la controladora SMC42. La señal Clock se configura inicialmente con un pulso de $10 \mathrm{~ms}$. La señal Direction se configura en un inicio a nivel bajo, correspondiente al cierre de la pinza, y siendo nivel alto cuando se precise su apertura. Por último, la señal Enable se inicializa a nivel alto. 


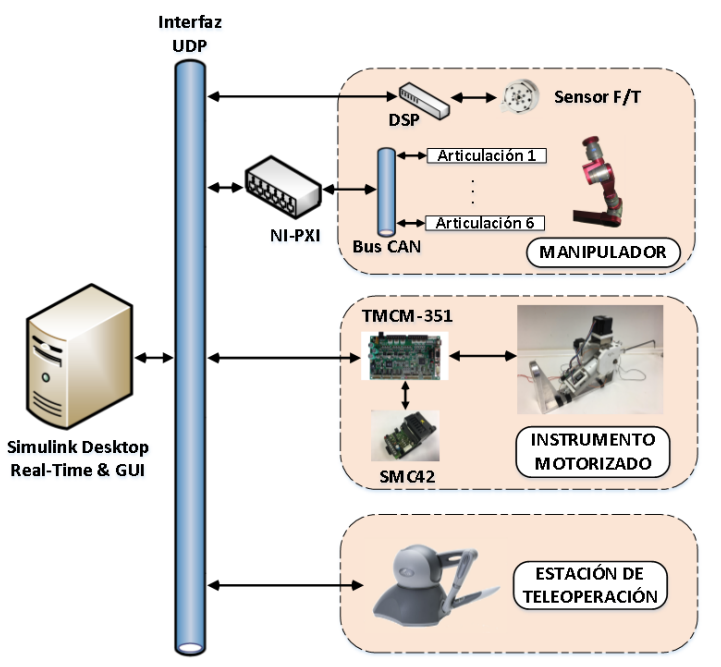

Figura 6: Arquitectura de la plataforma CISOBOT.

\section{ENTORNO DE TELEOPERACIÓN}

la figura 6 muestra la arquitectura del sistema de teleoperación que se ha empleado en la plataforma CISOBOT. Esta arquitectura consta de un PC que emplea el software Simulink Desktop Realtime para ejecutar el programa de control. Este PC se comunica con tres sistemas: manipulador, instrumento motorizado y estación de teleoperación. Primero, el sistema del manipulador compuesto por un sensor de esfuerzos y un brazo robótico. El segundo, es el instrumento motorizado que incluye la propia herramienta y las dos controladoras encargadas de su movimiento. Por último, el sistema de la estación de teleoperación está compuesto por un dispositivo háptico. Todas las comunicaciones entre los diferentes sistemas se realizan a través de un interfaz UDP.

La figura 7 se presenta la plataforma de teleoperación CISOBOT con la integración del instrumento motorizado.

\subsection{MANIPULADOR}

El robot esclavo de este sistema teleoperado se trata de un brazo robótico modular de seis grados de libertad, diseñado por la empresa Robotnik Automation mostrado en la figura 7. Este robot está basado en módulos rotativos PowerCube de la marca Schunk que funcionan como controladores distribuidos.

El envío y lectura de las medidas del manipulador se llevan a cabo a través de un programa desarrollado en LabVIEW e implantado en un ordenador embebido de tiempo real NI-PXI. Este programa se encarga de la inicialización de los manip-

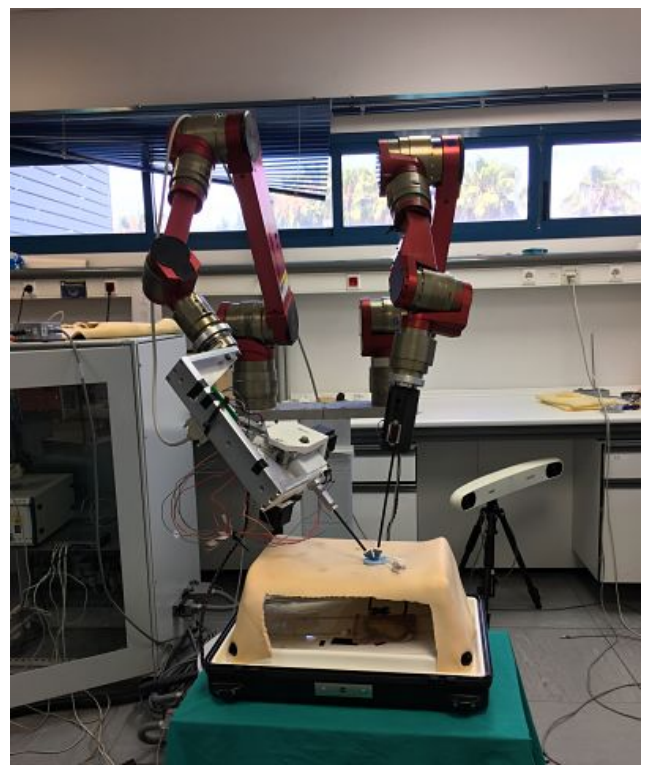

Figura 7: Plataforma CISOBOT dotada del instrumento motorizado.

uladores, y del envío de las referencias de velocidad o posición a las controladoras de cada articulación del manipulador. De igual forma recibe las posiciones y velocidades de cada articulación.

En la zona de interfaz entre el efector final del manipulador Robotnik y el instrumento motorizado se encuentra un sensor de esfuerzos fabricado por la empresa ATI Industrial Automation, en particular el modelo Network Force/Torque Sensor System o Net $F / T$. El dipositivo de control del sensor (DSP) proporciona un interfaz UDP, que ha permitido que este sensor se conecte directamente con la aplicación que se ejecuta en el PC.

\subsection{DISPOSITIVO HÁPTICO}

La estación de teleoperación está basada en una pantalla que muestra la imagen del interior del abdomen, así como dos dispositivos hápticos Phantom Omni. Estos dispositivo ofrecen hasta seis grados de libertad y proporciona retroalimentación de fuerzas de hasta $3.3 \mathrm{~N}$. La tabla 4 resume las características principales del dispositivo háptico empleado.

Tabla 4: Características del dispositivo háptico

\begin{tabular}{|c|l|}
\hline Especificación & Descripción \\
\hline Espacio de trabajo & $160 \times 120 \times 70 \mathrm{~mm}$ \\
Área de la base & $168 \times 203 \mathrm{~mm}$ \\
& $1.26 \mathrm{~N} / \mathrm{mm}$ Eje X \\
Rigidez & $2.31 \mathrm{~N} / \mathrm{mm} \mathrm{Eje} \mathrm{Y}$ \\
& $1.02 \mathrm{~N} / \mathrm{mm} \mathrm{Eje} \mathrm{Z}$ \\
\hline
\end{tabular}

El dispositivo háptico proprociona dos parámetros 


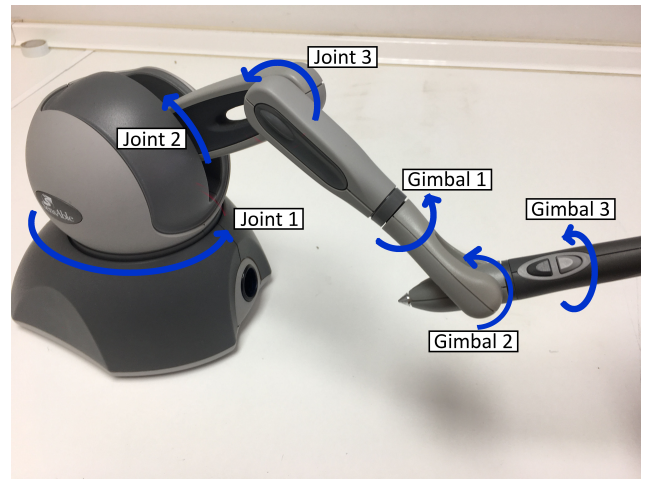

Figura 8: Aritculaciones del dispositivo háptico Phantom Omni.

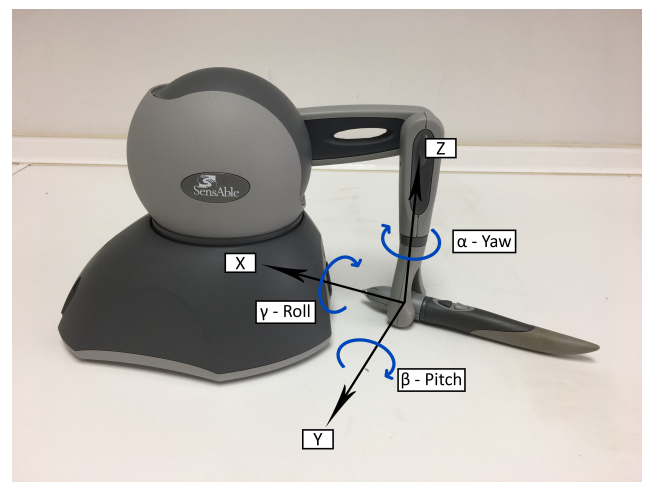

Figura 9: Representación de la orientación del lápiz del dispositivo háptico.

denominados Joints y Gimbals para conocer la posición y orientación del lápiz que permite realizar los movimientos. Como se puede ver en la figura 8, los tres primeros grados de libertad vienen dados por los denominados Joints, mientras que los tres siguientes son los denominados Gimbals. Estos últimos serán los utilizados para el cálculo de la orientación. La orientación del dispositivo háptico que se obtiene de dicho parámetro se corresponden con Roll, Pitch, Yaw como se muestra en la Figura 9.

Por otra parte, este dispositivo también consta de dos botones en el lápiz. El primer botón se emplea para activar los movimientos, es decir, el ma-
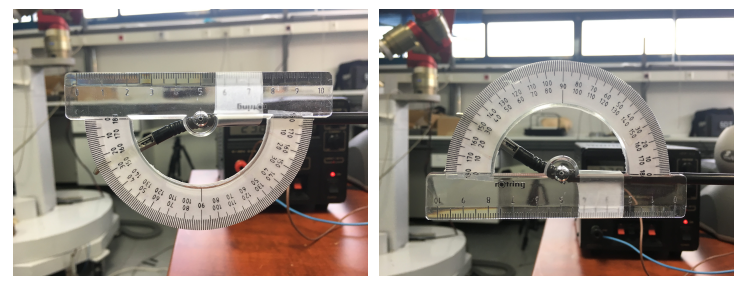

Figura 10: Ejemplo de medida de la posición angular en la punta de instrumento. nipulador y el instrumento se moverán mientras se mantenga pulsado el primer botón. El segundo botón se emplea para accionar el motor encargado de abrir y cerrar la pinza.

Con respecto a la comunicaciónes, este dispositivo consta de interfaz IEEE-1394 FireWire con la que se conecta directamente al PC. Debido a las limitaciones al emplear el entorno Simulink, las comunicaciones entre el sistema de control en Simulink y la estación de teleoperación se llevan a cabo a través de una aplicación desarrollada que hace de pasarela entre el dispositivo háptico y el PC. Estas aplicación se basa en la librería OpenHaptic, ofrecida por el fabricante.

\section{EXPERIMENTOS}

En esta sección se detalla el experimento realizado sobre el prototipo del instrumento motorizado, el cuál tiene como objetivo representar la relación motor-instrumento como se ha explicado anteriormente.

Para realizar este experimento se envían consignas de ángulos al motor. Empleando un transportador de ángulos en la punta (Figura 10) se comprueba posición angular de la punta del instrumento. Este procedimiento se ha repetido cada $5^{\circ}$ en un rango de ángulos entre $-45^{\circ}$ y $45^{\circ}$.

Las figuras 11 y 12 muestran los resultados obtenidos. Como se puede apreciar, el comportamiento de la punta de instrumento es bastante lineal en los extremos, sin embargo, se puede apreciar una pequeña zona muerta cuando el instrumento se mueve alrededor de los $0^{\circ}$.

\section{CONCLUSIONES}

Este artículo ha presentado los nuevos desarrollos llevados a cabo en la plataforma experimental CISOBOT. En concreto, se ha ampliado esta plataforma añadiéndole un instrumento motorizado capaz de mover su punta mediante teleoperación. Los resultados muestran que la relación de movimientos de los motores del instrumento no se corresponden directamente con el movimiento que se realiza en la punta. Por este motivo se ha realizado un experimento en el que se ha obtenido este relación para cada uno de los motores empleados. Por último, el instrumento ha quedado integrado en la plataforma CISOBOT. El siguiente paso es el desarrollo de algoritmos que permitan la realimentación de fuerzas, así como proporcionar guiado háptico al operador. Agradecimientos

A la Universidad de Málaga por el apoyo financiero del proyecto. 


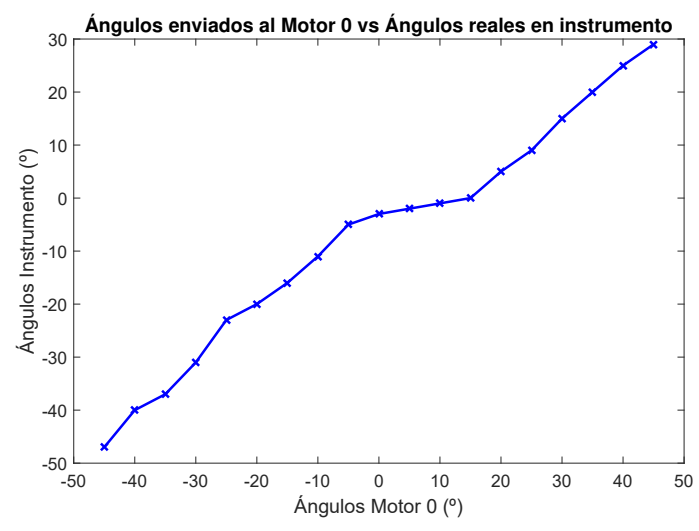

Figura 11: Relación entre la posición angular vertical del motor paso a paso (Motor 0) y la punta del instrumento.

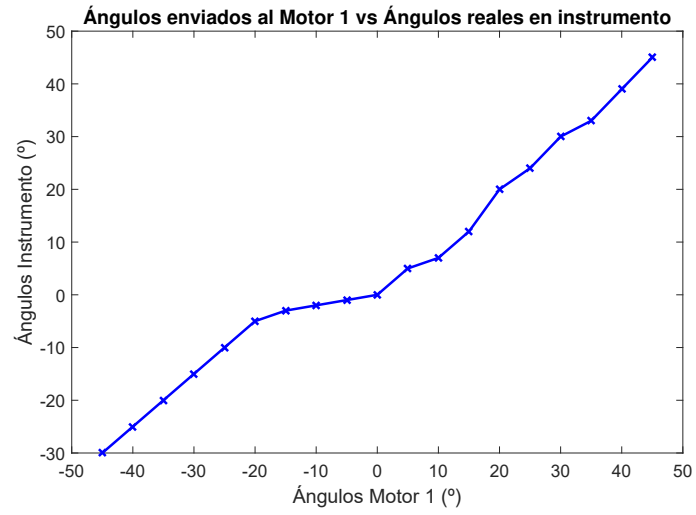

Figura 12: Relación entre la posición angular horizontal del motor paso a paso (Motor 1) y la punta del instrumento.

\section{Referencias}

[1] Alzugaray López, Ignacio, Teleoperación de manipuladores robóticos, (2014) Universidad de Málaga.

[2] Bauzano, E., Estebanez, B., Garcia-Morales, I., Muñoz, V. F., (2011) Robot quirúrgico auto-guiado para cirugía mínimamente invasiva en solitario. In Proc. Conf. RobotWorkshop, pp. 1-18.

[3] Beasley, R. A, (2012) Medical robots: current systems and research directions, Journal of Robotics.

[4] Casals, A., Frigola, M., Amat, J., (2009) La Robótica, una valiosa herramienta en Cirugía, Revista Iberoamericana de Automática e Informática Industrial RIAI, vol. 6, pp. 5-19.

[5] Chaparro Velasco, M. C. C., Vivas Albán, Ó A., (2016) Robótica quirúrgica, desde los grandes asistentes hasta la nanotecnología, Scientia et technica, vol. 21, pp. 182-190.

[6] Correa A. C., (2016) Sistemas robóticos teleoperados, Ciencia e Ingenier Ãa Neogranadina, vol.17 pp. 62-72.

[7] del Pulgar, C. P., Garcia-Morales, I., Blanco, I. R., Munoz, V. F., (2016) Navigation Method for Teleoperated Single-Port Access Surgery With Soft Tissue Interaction Detection, IEEE Systems Journal.

[8] del Pulgar, C. P., Garcia-Morales, López Casado M.C., Munoz, V. F., (2017) Navegación del instrumental en Robótica Quirúrgica, Jornadas Nacionales de Robótica, Spanish Robotics Conference.

[9] Gomes, P., (2011) Surgical robotics: Reviewing the past, analysing the present, imagining the future, Robotics and ComputerIntegrated Manufacturin, Elsevier.

[10] Hoeckelmann, M., Rudas, I. J, Fiorini, P., Kirchner, F., Haidegger, T., (2015) "Current capabilities and development potential in surgical robotic, International Journal of Advanced Robotic Systems, SAGE Publications Sage UK: London, England.

[11] Okamura, A. M, Mataric, M. J, Christensen, H. I, (2010) Surgical robotics: Medical and health-care robotics, IEEE Robotics \& Automation Magazine, vol. 17, pp. 23-37.

[12] Okamura, A. M, (2004) Methods for haptic feedback in teleoperated robot-assisted surgery, Industrial Robot: An International Journal, vol.31 pp. 499-508.

[13] Sanchez-Tapia, E. J., (2012) Implementación de un sistema teleoperado con reflexión de fuerza de seis grados de libertad. 